\title{
Poor Sleep in Patients with Multiple Sclerosis
}

\author{
Hanne Marie Bøe Lunde ${ }^{1 *}$, Tommy F. Aae ${ }^{1,2}$, William Indrevåg ${ }^{1,2}$, Jan Aarseth ${ }^{1}$, Bjørn Bjorvatn ${ }^{3,4}$, Kjell- \\ Morten Myhr ${ }^{1,2}$, Lars Bø ${ }^{1,2}$
}

1 The Norwegian Multiple Sclerosis Competence Centre, Department of Neurology, Haukeland University Hospital, Bergen, Norway, 2 KG Jebsen Centre for MS-Research, Department of Clinical Medicine, University of Bergen, Bergen, Norway, 3 Norwegian Competence Center for Sleep Disorders, Haukeland University Hospital, Bergen, Norway, 4 Department of Public Health and Primary Health Care, University of Bergen, Bergen, Norway

\begin{abstract}
Background: Poor sleep is a frequent symptom in patients with multiple sclerosis (MS). Sleep may be influenced by MSrelated symptoms and adverse effects from immunotherapy and symptomatic medications. We aimed to study the prevalence of poor sleep and the influence of socio-demographic and clinical factors on sleep quality in MS- patients.

Methods: A total of 90 MS patients and 108 sex-and age- matched controls were included in a questionnaire survey. Sleep complaints were evaluated by Pittsburgh Sleep Quality Index (PSQI) and a global PSQI score was used to separate good sleepers $(\leq 5)$ from poor sleepers $(>5)$. Excessive daytime sleepiness, the use of immunotherapy and antidepressant drugs, symptoms of pain, depression, fatigue and MS-specific health related quality of life were registered. Results were compared between patients and controls and between good and poor sleepers among MS patients.

Results: MS patients reported a higher mean global PSQI score than controls (8.6 vs. 6.3, $p=0.001)$, and $67.1 \%$ of the MS patients compared to $43.9 \%$ of the controls $(p=0.002)$ were poor sleepers. Pain $(p=0.02)$, fatigue $(p=0.001)$, depression $(p=0.01)$ and female gender $(p=0.04)$ were associated with sleep disturbance. Multivariate analyses showed that female gender $(p=0.02)$, use of immunotherapy $(p=005)$ and a high psychological burden of MS $(p=0.001)$ were associated with poor sleep among MS patients.
\end{abstract}

Conclusions: Poor sleep is common in patients with MS. Early identification and treatment of modifiable risk factors may improve sleep and quality of life in MS.

Citation: Bøe Lunde HM, Aae TF, Indrevåg W, Aarseth J, Bjorvatn B, et al. (2012) Poor Sleep in Patients with Multiple Sclerosis. PLoS ONE 7(11): e49996. doi:10.1371/journal.pone.0049996

Editor: Friedemann Paul, Charité University Medicine Berlin, Germany

Received June 7, 2012; Accepted October 18, 2012; Published November 14, 2012

Copyright: (c) 2012 Bøe Lunde et al. This is an open-access article distributed under the terms of the Creative Commons Attribution License, which permits unrestricted use, distribution, and reproduction in any medium, provided the original author and source are credited.

Funding: This research was funded by the Norwegian Multiple Sclerosis Competence Centre, Bergen, Norway; and unrestricted grants from Novartis Norway. The funders had no role in study design, data collection and analysis, decision to publish, or preparation of the manuscript.

Competing Interests: The authors have declared that no competing interests exist.

*E-mail: hmbl75@gmail.com

\section{Introduction}

Patients with multiple sclerosis (MS) frequently report poor sleep, and sleep disorders are more common in MS patients compared to healthy controls [1]. Causes of poor sleep in MS are likely multifactorial, and potential triggers may relate to adverse effects from immunotherapy and symptomatic medications and to MS-associated symptoms, such as pain and fatigue [2]. Certain symptoms related to MS, may lead to exacerbation of others if left untreated [3]. Patients suffering from a sleep disturbance have an increased risk of developing co-morbid conditions like heart disease, obesity and diabetes that may have a profound impact on long-term health $[4,5]$. In order to improve sleep and possibly reduce long-term health consequences of poor sleep in MS, identification of modifying risk factors of poor sleep is needed. Therefore we aimed to study the prevalence of sleep disturbance in MS, compared to the healthy Norwegian population. Further, among MS-patients, we aimed to identify possible socio-demographic and clinical factors associated with the risk of being a poor sleeper. Two prior studies have described the socio-demographic and clinical factors associated with poor sleep, both lacking a control group for comparisons $[1,6]$. To our knowledge, a case- control study looking at the socio-demographic and clinical associations with poor sleep in MS, has not been published previously.

\section{Materials and Methods}

\section{Ethics statement}

The study was based on written informed consent, and approved by the Regional Committee for Medical and Health Research Ethics of Western Norway.

\section{Patients and controls}

We aimed to include patients' with well-established disease. All 140 patients diagnosed with MS from 1993-1998 at the Department of Neurology, Haukeland University Hospital, Bergen, Norway were invited to participate in the study. All MS-patients fulfilling the diagnostic criteria of Poser were included, independent of subtype. The patients were identified though hospital records and recruited at the Department of Neurology, Haukeland University Hospital. A total of 280 ageand gender-matched controls were drawn from the Norwegian 
National Population Registry. There were no exclusion criteria. The data were collected by use of validated self-administered questionnaires and returned by mail in pre-stamped addressed return envelopes. All participants received the same set of study information and questionnaires, except for the MSIS-29 questionnaire, specifically designed for MS patients. A reminder was sent to each non-respondent participant via the mail two months after the initial questionnaires were mailed out. The collected data from the questionnaires were registered and entered in a database that was carefully double and triple checked for any mistakes in data entry.

\section{Variables}

Age, gender, marital status and the use of medication were registered. The regular medications were recorded and grouped into three classes: antidepressant drugs, immunotherapy and others. The questionnaires used are all validated, and all but the VAS pain scale are also validated in Norwegian [7-11].

Pittsburgh Sleep Quality Index (PSQI) was included to measure sleep and identify sleep complaints during the previous month [12]. It has several clinical and research applications and can be used to separate "good" from "poor" sleepers, screen for night time sleep disturbances and to monitor the progression of sleep disorders $[13,14]$. However, it cannot be used to diagnose specific sleep disorders and does not provide information on sleep stages or sleep architecture that can be measured by polysomnography (PSG) [15]. It consists of 19 self- rated questionnaires and 5 additional questionnaires for bed partner.

It provides a global score of sleep on a scale from 1 to 21, with higher scores indicating more sleep complaints. A global PSQI score $>5$ is shown to have a diagnostic sensitivity of $89.6 \%$ and a specificity of $86.5 \%$ and was used to separate good sleepers $(\leq 5)$ from poor sleepers $(>5)$ [12]. Test-retest reliability has shown consistency over time [16].MS-specific health related quality of life was recorded by the Multiple Sclerosis Impact Scale (MSIS-29), that includes the physical (20 items) and psychological (9 items) impact of MS. High scores on MSIS-29 indicate greater impact of MS. Naturally, only MS patients answered these questions, and associations to poor sleep was analysed separately for the physiological and psychological subscales of MSIS-29 (Hobert J) [17].

Excessive daytime sleepiness was registered by the Epworth sleepiness scale (ESS), that is a validated and widely used selfadministered questionnaire used to investigate excessive daytime sleepiness/hypersomnia [18]. It can be used as a screening test for excessive sleepiness or longitudinally to follow a patient's response to an intervention. It measures the proneness to fall asleep or doze off in eight specific situations. An ESS score above 10 is regarded as an indicator of excessive sleepiness.

We assessed acute pain by using the validated Visual Analogue Scale for pain assessment. The VAS pain scale consists of a $10 \mathrm{~cm}$ (3.94 inches) line with one end labelled "no pain" and the other end labelled "worst imaginable pain ever". The participants were asked to mark the line at the point that best described the pain intensity. For qualitative analysis we separated the data in two groups "no pain (0-1 points)" and "pain ( $>1$ point) [19]. The presence of pain at the time of completing the questionnaire was registered as present or not [20].

Fatigue was registered by the Fatigue Questionnaire (FQ) that is a validated questionnaire used to assess fatigue severity. It is an 11 item questionnaire used to measure physical and mental fatigue during the previous month (scale 0 -3) with a cut off score at 4 considered indicative of excessive fatigue [21].
Symptoms of depression were screened by the Beck Depression Inventory (BDI-II) that includes 21-question self-reporting multiple choice questions. The patients BDI score was categorized as not depressed (score 0-19) and depressed (score>20) [11,22].

\section{Statistical analysis}

Socio-demographic variables (age, gender, and marital status), medication, and clinical characteristics as well as the seven components of the PSQI questionnaire were compared between patients and controls. Among MS patients, the same variables in addition to health related quality of life (MSIS-29) was compared between good- and poor sleepers.

The Fischer Exact Test was applied to compare groups for nominal variables. The Mantel-Haenszel test was used for ordinal variables and independent two sample t- test was applied for continuous variables. Normal distribution and equality of variance was checked before applying the parametric t-test. Crude p-values are reported.

Independent two sample t- test was applied for continuous variables. Normal distribution and equality of variance was checked before applying the parametric t-test. Crude p-values are reported.

An analysis was performed to identify factors associated with poor sleep. Logistic regression was performed to estimate odds ratios, first in univariate models until a multivariate model was fitted. Variables with univariate $P<0.25$ were selected as candidates in the multivariate analysis following a model-building strategy as described by Hosmer \& Lemeshow [23].

Data analyses were carried out using SPSS software for Windows (version 15.0, SPSS, Chicago IL), p-values $<0.05$ were considered statistically significant.

\section{Results}

\section{Comparisons of patients and controls}

A total of $90 \mathrm{MS}$ patients $(64.3 \%)$ and $108(38.6 \%)$ control persons responded to the invitation to participate in the survey. Socio-demographic characteristics and the use of antidepressant drugs did not differ significantly among patients and controls (Table 1). The average age of the patients were $45 \pm 10.4$ and the controls were $44.3 \pm 10.2(p=0.65)$. Of the patients, $41(45.6 \%)$ were men and $49(54.4 \%)$ were women, and $45(41.7 \%)$ men and $63(58.3 \%)$ women made up the control group $(\mathrm{p}=0.667)$ (Table 1$)$. The mean score of excessive daytime sleepiness (ESS) did not differ between patients and controls and mean values were below cut-off for excessive sleepiness (data not shown). Pain $(p=0.01)$, fatigue $(p=0.004)$ and depression $(p=0.02)$ were reported more frequently among patients than controls. Thirty-five $(42.7 \%)$ patients received immunotherapy (interferon beta $(n=24)$, glatiramer acetate $(\mathrm{n}=7)$, natalizumab $(\mathrm{n}=2))$ (Table 1$)$. MS patients had a

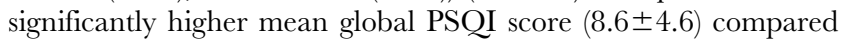
to controls $(6.3 \pm 4.1)$, indicating a poorer sleep quality $(p=0.001)$ (Table 2). PSQI sleep onset latency was significantly higher $(1.4 \pm 1.1)$ among patients than controls $(1.1 \pm 1.1)(0.04)$ (Table 2). The proportion of MS patients $(67.1 \%)$ that reported poor quality of sleep was significantly higher than the controls $(43.9 \%)$ $(\mathrm{p}=0.002)$.

\section{Risk factors associated with poor sleep among patients with MS}

More women $(78.0 \%)$ than men $(53.1 \%)$ suffered from poor sleep among the MS patients $(p=0.04)$. Further, pain $(p=0.02)$ and fatigue $(p=0.001)$ were associated with higher risk for being a poor sleeper (Table 3). All patients classified with moderate 
Table 1. Clinical and socio-demographic parameters among patients and controls.

\begin{tabular}{|c|c|c|c|}
\hline \multirow[t]{2}{*}{ Variable } & \multirow{2}{*}{$\begin{array}{l}\text { Patients } \\
\mathrm{N}=90^{*}\end{array}$} & \multirow{2}{*}{$\begin{array}{l}\text { Controls } \\
\mathrm{N}=108^{* *}\end{array}$} & \multirow[t]{2}{*}{ p-value } \\
\hline & & & \\
\hline Age, yrs $($ mean $\pm S D)$ & $45.0 \pm 10.4$ & $44.3 \pm 10.2$ & 0.65 \\
\hline Gender (female; \%) & 54.4 & 58.3 & 0.67 \\
\hline Married (\%) & 58.5 & 66.7 & 0.29 \\
\hline Antidepressant drugs (\%) & 9.8 & 3.7 & 0.08 \\
\hline Immunotherapy (\%) & 42.7 & 1.9 & $<0.001$ \\
\hline Excessive daytime sleepiness (ESS) (\%) & 33.8 & 22.6 & 0.10 \\
\hline Pain (VAS) (\%) & 38.8 & 21.3 & 0.01 \\
\hline Fatigue (FQ) (\%) & 35.5 & 15.8 & 0.004 \\
\hline Depression (BDI) (\%) & 15.8 & 4.2 & 0.02 \\
\hline MSIS-29 physiological disease severity & $27.4 \pm 24.7$ & NA & - \\
\hline MSIS-29 psychological disease severity & $28.1 \pm 25.1$ & NA & - \\
\hline \multicolumn{4}{|c|}{$\begin{array}{l}\text { ESS = Epworth Sleepiness Scale; FQ = Fatigue Questionnaire; BDI = Beck. } \\
\text { Depression Inventory; MSIS-29= Multiple Sclerosis Impact Scale. } \\
\text { *Number of patients range from 76-90; } \\
\text { **Number of controls range from } 96-108 . \text { Immunotherapy (IFNB, glatiramer } \\
\text { acetate, natalizumab). } \\
\text { doi:10.1371/journal.pone.0049996.t001 }\end{array}$} \\
\hline
\end{tabular}

depression $(p=0.01)$ and all patients on antidepressant medications suffered from poor sleep $(p=0.03)$. Excessive daytime sleepiness was not significantly associated with poor sleep among patients $(p=0.12)$ (Table 3). Higher score on mean MSIS-29 physiological and psychological sub scores, were associated with higher risk of being a poor sleeper $(\mathrm{p}<0.001)$.

Multivariate logistical regression analyses showed that female gender, $\quad(\mathrm{OR}=6.8, \quad 95 \% \quad \mathrm{CI}: \quad 1.33-35.3), \quad$ immunotherapy $(\mathrm{OR}=4.7,95 \%$ CI: $0.98-23.3)$ and reduced quality of life (MSIS-29 psychological subscale) $(\mathrm{OR}=1.12,95 \%$ CI: $1.05-$ 1.19) were associated with poor sleep (Table 4).

\section{Discussion}

Poor sleep is a common finding in MS patients, and previous studies have indicated a prevalence of $50 \%$ in this patient group $[24,25]$. In the present survey, we report a higher frequency of MS patients $(67.1 \%)$ with disturbed sleep. The focus of this study was to disclose potential modifiable clinical and socio-demographic (age, gender, marital status) risk factors associated with sleep disturbance in MS patients. Among demographic variables that were associated with sleep disturbance, female gender was found to be strongly associated with poor sleep. This is in contrast to a previous study that reported no influence of gender on sleep quality among MS patients [1].

Psychiatric disorders, and especially depression, are prevalent in MS and have major impact on quality of life [26].We found a 3fold higher prevalence of depressive symptoms among patients compared to controls, consistent with the results of a previous study [27]. All patients classified as having moderate depression in our study suffered from poor sleep. Insomnia and depression are closely linked, and appear to have a bidirectional relationship where insomnia worsens depression and vice versa [28,29]. MS patients reporting poor sleep should therefore be screened for depression, and treated accordingly.
Table 2. Pittsburgh Sleep Quality Index (PSQI) scores among patients and controls.

\begin{tabular}{|c|c|c|c|}
\hline \multirow[t]{2}{*}{ Variable* } & \multirow{2}{*}{$\begin{array}{l}\text { Patients } \\
\mathrm{N}=90^{* *}\end{array}$} & \multirow{2}{*}{$\begin{array}{l}\text { Controls } \\
\mathrm{N}=108^{* * *}\end{array}$} & \multirow[t]{2}{*}{$p$-value } \\
\hline & & & \\
\hline PSQI global score & $8.6 \pm 4.6$ & $6.3 \pm 4.1$ & 0.001 \\
\hline PSQI sleep quality & $1.1 \pm 0.7$ & $0.9 \pm 0.8$ & 0.03 \\
\hline PSQI sleep onset latency & $1.4 \pm 1.1$ & $1.1 \pm 1.1$ & 0.04 \\
\hline PSQI sleep duration & $0.9 \pm 0.8$ & $0.8 \pm 0.7$ & 0.39 \\
\hline PSQI sleep efficiency & $0.7 \pm 1.0$ & $0.5 \pm 0.8$ & 0.10 \\
\hline PSQI sleep disturbance & $1.4 \pm 0.6$ & $1.3 \pm 0.6$ & 0.33 \\
\hline PSQI hypnotic drugs & $0.7 \pm 1.2$ & $0.3 \pm 0.8$ & 0.005 \\
\hline PSQI daytime dysfunction & $1.3 \pm 0.8$ & $0.8 \pm 0.7$ & $<0.001$ \\
\hline
\end{tabular}

*Data are displayed as mean standard deviation;

**Number of patients range from 76-90;

***Number of controls range from 96-108.

doi:10.1371/journal.pone.0049996.t002

Table 3. Comparisons of clinical and socio-demographic parameters in MS patients categorized as good sleepers versus poor sleepers.

\begin{tabular}{|c|c|c|c|}
\hline Variable & $\begin{array}{l}\text { Good } \\
\text { sleepers }\end{array}$ & $\begin{array}{l}\text { Poor } \\
\text { sleepers }\end{array}$ & $p$-value \\
\hline Age, Mean $\pm S D$ & $43.2 \pm 12.0$ & $46.2 \pm 10.4$ & 0.27 \\
\hline Gender, n (\%) & & & 0.04 \\
\hline Female & $9(22.0)$ & $32(78.0)$ & \\
\hline Male & $15(46.9)$ & $17(53.1)$ & \\
\hline Married, n (\%) & & & 0.80 \\
\hline No & $11(35.5)$ & $20(64.5)$ & \\
\hline Yes & $13(31.0)$ & $29(69.0)$ & \\
\hline Antidepressant drugs, n (\%) & & & 0.047 \\
\hline No & $24(36.9)$ & $41(63.1)$ & \\
\hline Yes & $0(0.0)$ & $8(100)$ & \\
\hline Immunotherapy, n (\%) & & & 0.21 \\
\hline No & $17(39.5)$ & $26(60.5)$ & \\
\hline Yes & $7(23.3)$ & $23(76.7)$ & \\
\hline ESS, n (\%) & & & 0.12 \\
\hline $0-10$ & $18(39.1)$ & $28(60.9)$ & \\
\hline Above 10 & $5(20.0)$ & $20(80.0)$ & \\
\hline Pain, n (\%) & & & 0.02 \\
\hline No & $19(44.2)$ & $24(55.8)$ & \\
\hline Yes & $5(17.2)$ & $24(82.8)$ & \\
\hline Fatigue (FQ), n (\%) & & & 0.001 \\
\hline $0-4$ & $21(46.7)$ & $24(53.3)$ & \\
\hline Above 4 & $2(8.0)$ & $23(92.0)$ & \\
\hline Depression (BDI II), n (\%) & & & 0.01 \\
\hline $0-19$ & $23(39.7)$ & $35(60.3)$ & \\
\hline Above 19 & $0(0.0)$ & $10(100)$ & \\
\hline MSIS-29 physiological, Mean \pm SD & $10.5 \pm 13.9$ & $34.9 \pm 22.1$ & $<0.001$ \\
\hline MSIS-29 psychological, Mean \pm SD & $9.4 \pm 12.9$ & $38.0 \pm 22.6$ & $<0.001$ \\
\hline
\end{tabular}

MSIS-29: Multiple Sclerosis Impact Scale. ESS: Excessive daytime sleepiness. FQ: Fatigue questionnaire, BDI: Beck Depression Inventory.

doi:10.1371/journal.pone.0049996.t003 
Table 4. Independent factors associated with poor sleep in patients with MS.

\begin{tabular}{|c|c|c|c|}
\hline Variable & Odds Ratio (OR) & $95 \% \mathrm{Cl}$ & $p$-value \\
\hline MSIS-29; psychological subscale & $1,12^{*}$ & $1.05-1.19$ & 0.001 \\
\hline Immunotherapy & 4.74 & $0.98-23.26$ & 0.054 \\
\hline Gender (female) & 6.86 & $1.33-35.25$ & 0.021 \\
\hline $\begin{array}{l}\text { MSIS-29= Multiple Sclerosis Impa } \\
{ }^{*} \text { OR }=1.12 \text { denotes OR for each i } \\
\text { psychological subscale. } \\
\text { doi: } 10.1371 / \text { journal.pone. } 0049996\end{array}$ & $\begin{array}{l}\text { ct Scale-29; } \\
\text { creasing point achie } \\
\text { t004 }\end{array}$ & ved in MSIS-2S & \\
\hline
\end{tabular}

Subjective sleep disorders in MS and in the general population have in numerous epidemiological studies showed a close a close association with symptoms of fatigue [1,30,31]. Sleep disturbance has been reported as a significant independent contributor to fatigue in MS, and in our study, as many as $92 \%$ of the patients suffering from fatigue was classified as poor sleepers. A recent randomized controlled therapy study revealed improvement in fatigue by treatment of sleep disorders in MS [32]. However, the pathophysiology of MS-related fatigue is poorly understood and the cause and effect relationship between disturbed sleep and MSrelated fatigue is currently an enigma.

Poor quality of sleep in MS patients was not associated with excessive daytime sleepiness, despite increased frequencies of fatigue and sleep complaints in patients categorized as poor sleepers. These results are compatible with a pupillographic and questionnaire (ESS,SSS) study of $61 \mathrm{MS}$ patients, that reported no evidence of excessive daytime sleepiness in MS [33]. Symptoms of fatigue and sleepiness in MS patients are often confused and used interchangeably [34]. Subjective sleepiness and subjective fatigue in MS patients and healthy controls have shown to be independently associated with sleep disorders, although correlated with each other [34]. Patients with insomnia often complain of fatigue, but not sleepiness or the propensity to fall asleep [34]. These studies suggest that fatigue and excessive daytime sleepiness are differentiated conditions and should be assessed independently in patients complaining of poor sleep.

Acute and chronic pain is a frequent symptom in MS, and include neuropathic pain and musculoskeletal pain secondary to other MS symptoms such as spasticity [35]. Population studies have estimated that $55-65 \%$ of MS patients suffer from acute, subacute or chronic pain syndrome [36]. We found that the presence of pain was reported about twice as frequent among MSpatients compared to controls. Pain was also significantly more frequent in poor sleepers than in good sleepers. Similar findings was reported in a previous study, that in addition found pain to be the main cause of initial insomnia [37]. In an epidemiologic study of women with RRMS, sleep disturbance intensity were higher for women with RRMS with pain than for women without pain [38]. Pain is a common, but often inadequately treated symptom in MS [39]. By modifying pain, improved sleep may be obtained.

Separate analyses of each PSQI component showed that increased sleep latency was the most frequent complaint among MS patients, followed by sleep disturbance and daytime dysfunction. This was in line with a previous study, where initial insomnia was rated as the most common complaint of the PSQI component values [1].

No significant differences were noted between patients and controls regarding sleep disturbance and sleep efficiency.

The results from our study reveal that the psychological and physiological impact of MS is greater among poor sleepers than among good sleepers. In our study a high psychological burden of MS was independently associated with poor sleep. Nearly half of MS patients have reported significant anxiety levels within the first year of diagnosis [40]. Traditionally, management of MS have focused primarily on treatment of motor symptoms [41]. Treatment of non-motor symptoms such as psychological impact of MS is usually associated with improvement of HRQoL [41]. Sleep disruption evident by polygraphic registration is associated with greater psychological impact on quality of life [42]. More emphasis on the relief of psychological distress of MS may likely favour good sleep.

In this study, the use of immunotherapy was independently associated with poor quality of sleep. An actigraphy study of relapsing-remitting MS patients using immunomodulatory (interferon beta, glatirmaer acetate) drugs, showed a reduction in sleep efficiency in two-thirds on the night following interferon beta injection, irrespective of the frequency of injections [43]. Another study recently reported reduced flu-like symptoms and improved sleep efficacy by switching from evening to morning injections of interferon beta [44].

It is a usual recommendation from neurologists, that interferon beta should be injected in the evening, so that the patient can sleep through the side-effects [45]. We have systematically advised patients to take evening injections of IFNB. Although our results reveal no causality between use of immunotherapy and poor sleep, we believe however, that our findings are sufficiently suggestive to introduce a possible theory on the relationship between timing of immunomodulatory drugs and impact on sleep. The possibility that improved sleep may be obtained by changing administration time of immunomodulatory medication, is important in regards to sleep management, and further studies should be warranted in this regard.

There are several limitations in our study. As this is a cross sectional study, we cannot determine the directions of the predictors of poor sleep. We can only show significant clinical and demographic associations to poor sleep quality. The lower response rate in the control group can be explained by the collection of information by mail, and by a set of questionnaires that was comprehensive and time demanding. Controls suffering from poor sleep may have had particular interest in this subject; this may have skewed the data towards smaller differences between patients and controls.

Micturition-related symptoms such as urge and nocturia were not analysed separately, as these parameters were included in the sleep quality component of MSIS -29 and PSQI respectively. Furthermore, the pain assessment was limited and multidimensional measures such as McGill Pain Questionnaire or Multidimensional pain inventory, would possibly have added more information. The expanded disability scale (EDSS) was not assessed, and therefore a possible relationship between disability status and sleep disturbance could not be established.

The results of this study indicate that poor sleep is a frequent complaint among patients with MS and independently associated with female gender, use of immunotherapy and a high psychological burden of MS. Sleep disturbance is a potentially treatable condition. Increased awareness of modifiable risk factors of poor sleep in MS may be important for early therapeutic and prophylactic interventions.

\section{Acknowledgments}

The authors would like to thank researchers Nina Grytten Torkildsen and Kristoffer Haugarvoll for helpful discussions regarding the manuscript. 


\section{Author Contributions}

Conceived and designed the experiments: HMBL TFA WI JA BB KMM LB. Performed the experiments: HMBL TFA WI JA BB KMM LB.

\section{References}

1. Merlino G, Fratticci L, Lenchig G, Valente M, Cargnelutti D, et al. (2009) Prevalence of 'poor sleep' among patients with multiple sclerosis: an independent predictor of mental and physical status. Sleep Med 10: 26-34.

2. Brass SD, Duquette P, Proulx-Therrien J, Auerbach S (2010) Sleep disorders in patients with multiple sclerosis. Sleep Med Rev 14: 121-129.

3. Crayton H, Heyman RA, Rossman HS (2004) A multimodal approach to managing the symptoms of multiple sclerosis. Neurology 63: S12-18.

4. Attarian H (2009) Importance of sleep in the quality of life of multiple sclerosis patients: a long under-recognized issue. Sleep Med 10: 7-8.

5. Kaminska M, Kimoff R, Benedetti A, Robinson A, Bar-Or A, et al. (2011) Obstructive sleep apnea is associated with fatigue in multiple sclerosis. Mult Scler.

6. Bamer AM, Johnson KL, Amtmann DA, Kraft GH (2010) Beyond fatigue: Assessing variables associated with sleep problems and use of sleep medications in multiple sclerosis. Clin Epidemiol 2010: 99-106.

7. Beiske KK, Kjelsberg FN, Ruud EA, Stavem K (2009) Reliability and validity of a Norwegian version of the Epworth sleepiness scale. Sleep and Breathing 13: 65-72.

8. Loge JH, Ekeberg O, Kaasa S (1998) Fatigue in the general Norwegian population: Normative data and associations. Journal of Psychosomatic Research 45: 53-65.

9. Smedal T, Johansen HH, Myhr KM, Strand LI (2010) Psychometric properties of a Norwegian version of Multiple Sclerosis Impact Scale (MSIS-29). Acta Neurologica Scandinavica 122: 244-251.

10. Pallesen S, Nordhus, I H., Omvik S., Sivertsen B., Matthiesen, S B., \& Bjorvatn B. (2005) Pittsburgh Sleep Quality Index. Tidsskrift for Norsk Psykologforening: $714-717$.

11. Aasen H (2001) An empirical investigation of depression symptoms: norms, psychometric characteristics and factor structure of the Beck Depression Inventory II Bergen.

12. Buysse DJ, Reynolds GF, 3rd, Monk TH, Berman SR, Kupfer DJ (1989) The Pittsburgh Sleep Quality Index: a new instrument for psychiatric practice and research. Psychiatry Res 28: 193-213.

13. Voderholzer U, Riemann D, Hornyak M, Backhaus J, Feige B, et al. (2001) A double-blind, randomized and placebo-controlled study on the polysomnographic withdrawal effects of zopiclone, zolpidem and triazolam in healthy subjects. Eur Arch Psychiatry Clin Neurosci 251: 117-123.

14. Hogl B, Arnulf I, Comella C, Ferreira J, Iranzo A, et al. (2010) Scales to assess sleep impairment in Parkinson's disease: critique and recommendations. Mov Disord 25: 2704-2716.

15. Dyken ME, Afifi AK, Lin-Dyken DC (2012) Sleep-related problems in neurologic diseases. Chest 141: 528-544.

16. Buysse DJ, Hall ML, Strollo PJ, Kamarck TW, Owens J, et al. (2008) Relationships between the Pittsburgh Sleep Quality Index (PSQI), Epworth Sleepiness Scale (ESS), and clinical/polysomnographic measures in a community sample. J Clin Sleep Med 4: 563-571.

17. Hobart J, Lamping D, Fitzpatrick R, Riazi A, Thompson A (2001) The Multiple Sclerosis Impact Scale (MSIS-29): a new patient-based outcome measure. Brain 124: $962-973$

18. Johns MW (1991) A new method for measuring daytime sleepiness: the Epworth sleepiness scale. Sleep 14: 540-545.

19. Collins SL, Moore RA, McQuay HJ (1997) The visual analogue pain intensity scale: what is moderate pain in millimetres? Pain 72: 95-97.

20. Kane RL, Bershadsky B, Rockwood T, Saleh K, Islam NC (2005) Visual Analog Scale pain reporting was standardized. J Clin Epidemiol 58: 618-623.

21. Chalder T, Berelowitz G, Pawlikowska T, Watts L, Wessely S, et al. (1993) Development of a fatigue scale. J Psychosom Res 37: 147-153.

22. Svanborg P, Asberg M (2001) A comparison between the Beck Depression Inventory (BDI) and the self-rating version of the Montgomery Asberg Depression Rating Scale (MADRS). J Affect Disord 64: 203-216.
Analyzed the data: HMBL TFA WI. Contributed reagents/materials/ analysis tools: HMBL TFA WI. Wrote the paper: HMBL TFA WI.

23. Hosmer DW LS (2000) Applied logistic regression, 2nd edn Wiley.

24. Tachibana N, Howard RS, Hirsch NP, Miller DH, Moseley IF, et al. (1994) Sleep problems in multiple sclerosis. Eur Neurol 34: 320-323.

25. Bamer AM, Johnson KL, Amtmann D, Kraft GH (2008) Prevalence of sleep problems in individuals with multiple sclerosis. Mult Scler 14: 1127-1130.

26. Lobentanz IS, Asenbaum S, Vass K, Sauter C, Klosch G, et al. (2004) Factors influencing quality of life in multiple sclerosis patients: disability, depressive mood, fatigue and sleep quality. Acta Neurol Scand 110: 6-13.

27. Clark CM, Fleming JA, Li D, Oger J, Klonoff H, et al. (1992) Sleep disturbance, depression, and lesion site in patients with multiple sclerosis. Arch Neurol 49: 641-643.

28. Paparrigopoulos T, Ferentinos P, Kouzoupis A, Koutsis G, Papadimitriou GN (2010) The neuropsychiatry of multiple sclerosis: focus on disorders of mood, affect and behaviour. Int Rev Psychiatry 22: 14-21.

29. Riemann D, Berger M, Voderholzer U (2001) Sleep and depression-results from psychobiological studies: an overview. Biol Psychol 57: 67-103.

30. Kaynak H, Altintas A, Kaynak D, Uyanik O, Saip S, et al. (2006) Fatigue and sleep disturbance in multiple sclerosis. Eur J Neurol 13: 1333-1339.

31. Merkelbach S, Schulz H, Fatigue Collaborative Study G (2006) What have fatigue and sleepiness in common? J Sleep Res 15: 105-106.

32. Cote I, Trojan D, Kaminska M, Cardoso M, Benedetti A, et al. (2012) Impact of sleep disorder treatment on fatigue in multiple sclerosis. Mult Scler.

33. Frauscher B, Egg R, Brandauer E, Ulmer H, Berger T, et al. (2005) Daytime sleepiness is not increased in mild to moderate multiple sclerosis: a pupillographic study. Sleep Med 6: 543-547.

34. Hossain JL, Ahmad P, Reinish LW, Kayumov L, Hossain NK, et al. (2005) Subjective fatigue and subjective sleepiness: two independent consequences of sleep disorders? J Sleep Res 14: 245-253.

35. Stenager E, Knudsen L, Jensen K (1995) Acute and chronic pain syndromes in multiple sclerosis. A 5-year follow-up study. Ital J Neurol Sci 16: 629-632.

36. O'Connor AB, Schwid SR, Herrmann DN, Markman JD, Dworkin RH (2008) Pain associated with multiple sclerosis: systematic review and proposed classification. Pain 137: 96-111.

37. Stanton BR, Barnes F, Silber E (2006) Sleep and fatigue in multiple sclerosis. Mult Scler 12: 481-486.

38. Newland PK, Naismith RT, Ullione M (2009) The impact of pain and other symptoms on quality of life in women with relapsing-remitting multiple sclerosis. J Neurosci Nurs 41: 322-328.

39. Wright LJ (2012) Identifying and treating pain caused by MS. J Clin Psychiatry 73: e23.

40. Janssens AC, van Doorn PA, de Boer JB, van der Meche FG, Passchier J, et al. (2003) Impact of recently diagnosed multiple sclerosis on quality of life, anxiety, depression and distress of patients and partners. Acta Neurol Scand 108: 389395.

41. Mitchell AJ, Benito-Leon J, Gonzalez JM, Rivera-Navarro J (2005) Quality of life and its assessment in multiple sclerosis: integrating physical and psychological components of wellbeing. Lancet Neurol 4: 556-566.

42. Trojan DA, Kaminska M, Bar-Or A, Benedetti A, Lapierre Y, et al. (2012) Polysomnographic measures of disturbed sleep are associated with reduced quality of life in multiple sclerosis. J Neurol Sci 316: 158-163.

43. Mendozzi L, Tronci F, Garegnani M, Pugnetti L (2010) Sleep disturbance and fatigue in mild relapsing remitting multiple sclerosis patients on chronic immunomodulant therapy: an actigraphic study. Mult Scler 16: 238-247.

44. Nadjar Y, Coutelas E, Prouteau P, Panzer F, Paquet D, et al. (2011) Injection of interferon-beta in the morning decreases flu-like syndrome in many patients with multiple sclerosis. Clin Neurol Neurosurg 113: 316-322.

45. Lublin FD, Whitaker JN, Eidelman BH, Miller AE, Arnason BG, et al. (1996) Management of patients receiving interferon beta-1b for multiple sclerosis: report of a consensus conference. Neurology 46: 12-18. 